\title{
1 FastMLST: A multi-core tool for 2 multilocus sequence typing of draft 3 genome assemblies
}

4 Enzo Guerrero-Araya ${ }^{1,2, *}$, Marina Muñoz ${ }^{1,2,3}$, César Rodríguez $^{3}$ and Daniel Paredes-Sabja ${ }^{1,2,5, *}$

$5{ }^{1}$ Microbiota-Host Interactions and Clostridia Research Group, Facultad de Ciencias de la Vida,

6 Universidad Andrés Bello, Santiago, Chile. ${ }^{2}$ ANID - Millennium Science Initiative Program -

7 Millennium Nucleus in the Biology of the Intestinal Microbiota, Santiago, Chile. ${ }^{3}$ Grupo de

8 Investigaciones Microbiológicas-UR (GIMUR), Departamento de Biología, Facultad de Ciencias

9 Naturales, Universidad del Rosario, Bogotá, Colombia. ${ }^{4}$ Facultad de Microbiología \& Centro de

10 Investigación en Enfermedades Tropicales (CIET), Universidad de Costa Rica, San José, Costa Rica. ${ }^{5}$

11 Department of Biology, Texas A\&M University, College Station, TX, 77843, USA.

$12 *$ To whom correspondence should be sent

13 Contact: Enzo Guerrero-Araya: enzoguerreroaraya@gmail.com and Daniel Paredes-Sabja:

14 dparedes-sabja@bio.tamu.edu 
Multilocus Sequence Typing (MLST) is a precise microbial typing approach at the intra-species level

17 for epidemiological and evolutionary purposes. It operates by assigning a sequence type (ST)

18 identifier to each specimen, based on a combination of allelic sequences obtained for multiple

19 housekeeping genes included in a defined scheme. The use of MLST has multiplied due to the

20 availability of large numbers of genomic sequences and epidemiological data in public

21 repositories. However, data processing speed has become problematic due to datasets' massive

22 size. Here, we present FastMLST, a tool that is designed to perform PubMLST searches using

23 BLASTn and a divide-and-conquer approach. Compared with mlst, CGE/MLST, MLSTar, and

24 PubMLST, FastMLST takes advantage of current multi-core computers to simultaneously type

25 thousands of genome assemblies in minutes, reducing processing times by at least 4-fold and with

26 more than $99.95 \%$ consistency.

27 Availability and Implementation: The source code, installation instructions and documentation

28 are available at https://github.com/EnzoAndree/FastMLST

\section{Abstract word count: 151}

\section{Introduction}

Multilocus Sequence Typing was a revolutionary attempt to standardize the molecular typing of

34 global relevance (Dingle, et al., 2001; Griffiths, et al., 2010; Martin-Rodriguez, et al., 2019; Meats, 
36 combination of alleles from an optimal set of housekeeping genes defined for each species. Even

37 though there are methods with higher discrimination power (cgMLST/wgMLST), MLST offers

38 several advantages, such as (1) a common language for typing, (2) simple implementation, and (3)

39 a vast amount of information collected throughout history (Kimura, 2018).

40 Currently available programs for ST determination from assembled genomes i.e. mlst (Seemann,

41 2015), CGE/MLST (Larsen, et al., 2012), MLSTar (Ferrés and Iraola, 2018), and the online tool

42 PubMLST (Jolley, et al., 2018) deliver results in 1-9 minutes when dealing with hundreds of

43 genomes. However, since most of them do not (or inefficiently) implement parallel computing,

44 processing of tens of thousands of genomes can be extremely time-consuming. In addition, their

45 outputs are typically restricted to a table with allelic profiles and do not deliver a file with

46 concatenated allele sequences necessary for subsequent phylogenetic analyses (Glaeser and

47 Kampfer, 2015).

48 In response to these limitations, we developed FastMLST, a tool focused on parallel computing via

49 a divide-and-conquer approach (Smith, 1985) that performs BLASTn searches (Camacho, et al.,

50 2009) against the sequences of MLST schemes deposited in the PubMLST database (Jolley, et al.,

51 2018). FastMLST delivers two main outputs: a table the allelic profiles detected for a genome

52 query and a multi-FASTA file containing their sequences.

\section{2. Methods}

\section{2.1. Implementation}

55 FastMLST is an open source standalone software implemented in Python 3 that only requires

56 BLASTn as an external program. FastMLST is easy to install via the Anaconda Package Manager

57 (https://anaconda.org/bioconda/fastmlst) and is in constant development. 


\section{$58 \quad$ 2.2. Key analysis steps}

\section{PubMLST database setup}

60 FastMLST can retrieve and update the PubMLST database (Jolley, et al., 2018) with its '--update-

61 mlst' option. It automatically processes the 150 currently available schemes (October 2020) and

62 prepares the system for subsequent deployment.

\section{Allele searches}

64 FastMLST is based on a divide-and-conquer approach, whereby each genome is processed in 65 parallel. Allele searches are performed with BLASTn using the public, regularly updated, and centralized database maintained at PubMLST (Jolley, et al., 2018).

\section{Scheme identification}

68 The MLST scheme to use for each query genome is inferred by an automatic scoring system,

69 similar to the one reported by (Seemann, 2015). This system assigns known, full-length alleles a maximum score of 100 points, $70 \%$ of the score to alleles of the expected length but with SNPS

71 (95\% identity by default), or $20 \%$ of the score to alleles with unexpected lengths. At the end, the

72 MLST scheme with the highest overall score is selected for further analyses. Alternatively, it is 73 possible to choose among schemes manually. When multiple perfect hits to a single scheme are

74 found, FastMLST reports all hits to alert the user of potential contaminations.

\section{3. Performance benchmark}

76 We assessed the capability of FastMLST to identify STs correctly for three bacterial species with

77 unequal numbers of scheme alleles. To this end, we measured the concordance over ST

78 assignment between FastMLST and PubMLST (Jolley, et al., 2018). The species tested were 
Cutibacterium acnes, Enterococcus faecium, and Streptococcus pneumoniae, which as of

80 September $11^{\text {th }} 2020$ included $153,1,833$ and 16,301 STs, respectively. Besides, we compared the

81 processing speeds of FastMLST and four others widely used software: mlst v2.11, CGE/MLST

82 V2.0.4, MLSTar v1.0, and the online tool PubMLST.

83 A total of 278 genomes for $C$. acnes, 1,915 for E. faecium, and 7,996 for S. pneumoniae obtained

84 from GenBank using ncbi-genome-download v0.2.11 (https://github.com/kblin/ncbi-genome-

85 download) were used in the analysis of ST assignment concordance (Supplementary Table 1).

86 Compared with PubMLST result (Supplementary Tables 2, 3 and 4), we show that FastMLST

87 (Supplementary Tables 5, 6 and 7) reaches a concordance of 99.96\% ( $n=9,502 / 9,506)($ Table 1). As

88 to the inconsistencies detected, FastMLST failed to assign STs to 4 genomes with multiple perfect

89 hits against the same locus, indicating that they are possibly contaminated (Supplementary Table 6

90 and 7). Additionally, FastMLST reported 401 STs that remained undetected by PubMLST (7 in $C$.

91 acnes, 216 in E. faecium, and 178 in S. pneumoniae) (Supplementary Table 5, 6 and 7). These new

92 ST are derived from new alleles or new combinations of known alleles (Table 1). On the other

93 hand, FastMLST missed some full-length alleles due to incomplete coverage $(<100 \%)$ (Table 1).

Table 1. Concordance analysis between FastMLST and PubMLST using $\mathbf{1 0 , 1 8 9}$ genomes.

\begin{tabular}{|c|c|c|c|c|c|c|c|}
\hline Dataset & Genomes & $\% G C$ & $\begin{array}{l}\text { ST assigned by } \\
\text { PubMLST }^{\text {a }}\end{array}$ & $\begin{array}{l}\text { ST assigned by } \\
\text { FastMLST }^{\text {bas }}\end{array}$ & Concordance $^{c}$ & \begin{tabular}{|lr} 
New ST \\
with full- \\
length \\
alleles $^{d}$
\end{tabular} & $\begin{array}{l}\text { Missing alleles } \\
(<100 \% \text { coverage })^{\mathrm{e}}\end{array}$ \\
\hline C. acnes & 278 & $60 \%$ & 259 & 259 & $259 / 259(100 \%)$ & 7 & 12 \\
\hline E. faecium & 1915 & $38 \%$ & 1545 & 1544 & $1544 / 1545$ (99.94\%) & 216 & 155 \\
\hline S. pneumoniae & 7996 & $39 \%$ & 7702 & 7699 & $7699 / 7702(99.96 \%)$ & 178 & 119 \\
\hline $\begin{array}{l}{ }^{a} \text { A genome was } \\
{ }^{b} \text { A genome was } \\
{ }^{c} \text { A ST assignme } \\
{ }^{d} \text { A genome witl } \\
{ }^{e} \text { Details of genc }\end{array}$ & $\begin{array}{l}\text { included in } \\
\text { included in } \\
\text { was consi } \\
\text { novel allele }\end{array}$ & $\begin{array}{l}\text { this ca } \\
\text { this ca } \\
\text { dered } \\
\text { s (100 }\end{array}$ & $\begin{array}{l}\text { tegory if an ST was } \\
\text { tegory if an ST was } \\
\text { concordant if the S } \\
\% \text { coverage). It is a }\end{array}$ & $\begin{array}{l}\text { assigned using } \mathrm{PL} \\
\text { assigned using } \mathrm{Fa} \\
\mathrm{T} \text { assigned by } \mathrm{Pub} \\
\text { designation only }\end{array}$ & $\begin{array}{l}\text { IbMLST. } \\
\text { MLST and FastMLST w } \\
\text { present in FastMLST. }\end{array}$ & sidentical. & \\
\hline
\end{tabular}


95 The speed of FastMLST, MLSTar, PubMLST and mlst (expressed in genomes/minute) was

96 compared in a workstation equipped with 2 Intel(R) Xeon(R) CPU E5-2683 v4 @ 2.10GHz using 1, 2,

$974,8,16,32$ or 64 cores. Each program was run in triplicate with a sample of 278 C. acnes genomes

98 each time. The results for PubMLST were obtained remotely because it does not offer a

99 standalone version. When a single core was used, mlst v2.11 achieved the best performance, with

10093 genomes/minute (Figure 1A). However, mlst v2.11 did not speed up when its multiple

101 processing option was enabled (Figure 1A). MLSTar can also use multiple processors, but it is at

102 least 4-fold slower than FastMLST (Figure $1 \mathrm{~A}$ and 1B). FastMLST's processing speed grew

103 exponentially as more cores were deployed (Figure 1B), reaching a speed of 1,642

104 genomes/minute using 64 cores. This allows processing of 16,000 genomes in less than 10

105 minutes.

A

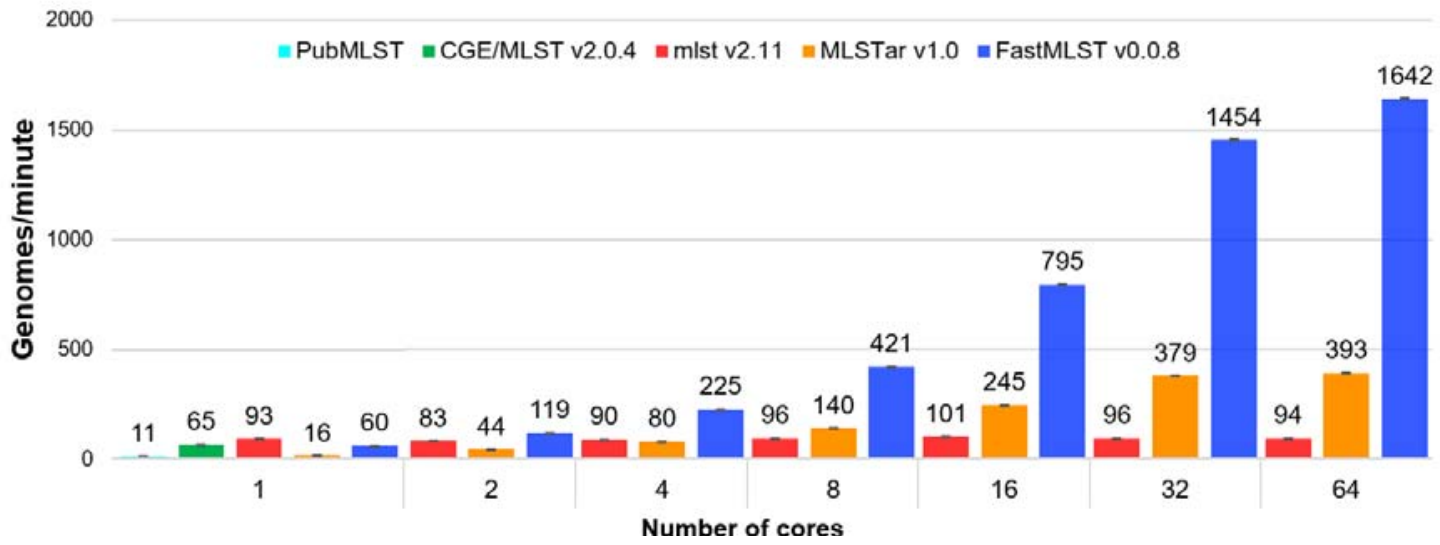

B

Parallel workflow

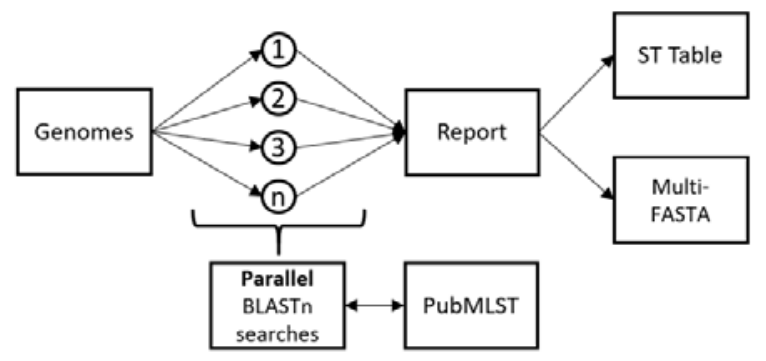

C

Serial workflow

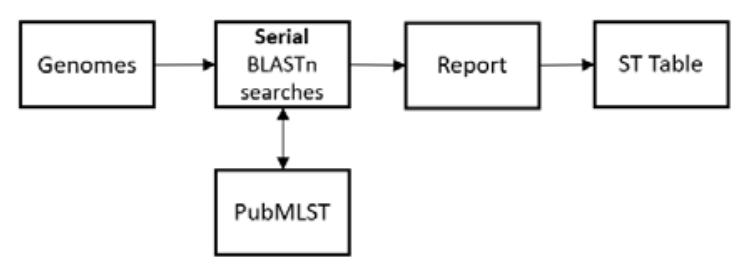


107 Figure 1. Processing speed and simplified comparative-workflow. (A) Processing speed

108 (genomes/minute) of FastMLST and standard software for MLST determination (mlst, PubMLST,

109 CGE/MLST and MLSTar) was compared by triplicate using a dataset composed of 278 C. acnes

110 genomes and 1, 2, 4, 8, 16, 32, and 64 cores. Error bars represent the standard error of three

111 replicates. Only mlst, MLSTar and FastMLST natively support parallel processing. (B) FastMLST and

112 MLSTar workflow. (C) mlst, PubMLST and CGE/MLST workflow.

113 4. Final remarks

114 FastMLST assigns STs to thousands of genomes in minutes with the additional plus of generating a

115 multi-FASTA file with concatenated allele sequences suitable for downstream phylogenetic

116 analysis. These unique features have the potential to boost future research.

\section{Funding}

118 This work was supported by a Doctoral fellowship 21181536 from ANID to E.G-A., and by EU-Lac

119 project "Genomic Epidemiology of Clostridium difficile in Latin America (T020076)", ANID -

120 Millennium Science Initiative Program - NCN17_093, and Start-up funds of Texas A\&M University

121 to D.P-S. 


\section{References}

123 Camacho, C., et al. BLAST+: architecture and applications. BMC Bioinformatics 2009;10:421.

124 Dingle, K.E., et al. Multilocus sequence typing system for Campylobacter jejuni. J Clin Microbiol

125 2001;39(1):14-23.

126 Ferrés, I. and Iraola, G. MLSTar: automatic multilocus sequence typing of bacterial genomes in R. 127 PeerJ 2018;6:e5098-e5098.

128 Glaeser, S.P. and Kampfer, P. Multilocus sequence analysis (MLSA) in prokaryotic taxonomy. Syst 129 Appl Microbiol 2015;38(4):237-245.

130 Griffiths, D., et al. Multilocus sequence typing of Clostridium difficile. J Clin Microbiol 131 2010;48(3):770-778.

132 Jolley, K.A., Bray, J.E. and Maiden, M.C.J. Open-access bacterial population genomics: BIGSdb 133 software, the PubMLST.org website and their applications. Wellcome Open Res 2018;3:124.

134 Kimura, B. Will the emergence of core genome MLST end the role of in silico MLST? Food Microbiol 135 2018;75:28-36.

136 Larsen, M.V., et al. Multilocus sequence typing of total-genome-sequenced bacteria. J Clin 137 Microbiol 2012;50(4):1355-1361.

138 Maiden, M.C., et al. Multilocus sequence typing: a portable approach to the identification of 139 clones within populations of pathogenic microorganisms. Proc Natl Acad Sci U $S$ A 140 1998;95(6):3140-3145.

141 Martin-Rodriguez, A.J., et al. Multilocus sequence typing of Shewanella algae isolates identifies 142 disease-causing Shewanella chilikensis strain 6I4. FEMS Microbiol Ecol 2019;95(1).

143 Meats, E., et al. Characterization of encapsulated and noncapsulated Haemophilus influenzae and 144 determination of phylogenetic relationships by multilocus sequence typing. $J$ Clin Microbiol 145 2003;41(4):1623-1636.

146 Seemann, T. 2015. mlst. https://github.com/tseemann/mlst

147 Smith, D.R. The design of divide and conquer algorithms. Science of Computer Programming $148 \quad 1985 ; 5: 37-58$. 